\title{
Fractal Modeling of Pore Structure and Ionic Diffusivity for Cement Paste
}

\author{
Yun Gao, ${ }^{1}$ Jin-yang Jiang, ${ }^{1}$ and Kai $\mathrm{Wu}^{2}$ \\ ${ }^{1}$ School of Materials Science and Engineering, Southeast University, Nanjing 211189, China \\ ${ }^{2}$ School of Materials Science and Engineering, Tongji University, Shanghai 201804, China \\ Correspondence should be addressed to Yun Gao; gaoyun3888@126.com
}

Received 27 July 2016; Revised 17 October 2016; Accepted 16 November 2016

Academic Editor: Kazunori Fujikake

Copyright (C) 2016 Yun Gao et al. This is an open access article distributed under the Creative Commons Attribution License, which permits unrestricted use, distribution, and reproduction in any medium, provided the original work is properly cited.

Pore structure in cement based composites is of paramount importance to ionic diffusivity. In this paper, pore structure in cement paste is modeled by means of the recently proposed solid mass fractal model. Moreover, an enhanced Maxwell homogenization method that incorporates the solid mass fractal model is proposed to determine the associated ionic diffusivity. Experiments are performed to validate the modeling, that is, mercury intrusion porosimetry and rapid chloride migration. Results indicate that modeling agrees well with those obtained from experiments.

\section{Introduction}

Ionic (i.e., chloride ion) diffusivity is one of the critical parameters in service life design of cement based composites for coastal infrastructures [1]. In recent years, much attention has been drawn to the direct assessment of ionic diffusivity on a basis of pore structure which could be obtained from experiment or modeling [2]. However, a practical challenge still exists as a result of the intrinsic complexity of cement based composites. In particular, the pore size distribution may range from nanometer (C-S-H gel pore) to micrometer (capillary pore) of several orders [3]. The multiscale approach is therefore an option, which deals with C-S-H gel and capillary pore in terms of the packing of basic globules and hydration products, respectively $[4,5]$. Nevertheless, as it has to assign a large number of parameters and assumptions, the multiscale approach often leads to substantial disadvantages in efficiency and viability. More robust approaches would be beneficial to describe the complex pore structure as well as ionic diffusivity for cement based composites.

The fractal characters have been recognized among natural and artificial porous materials such as limes, soils, rocks, and ceramics [6-10]. In essence, fractal describes a natural phenomenon or object that exhibits similar pattern at different scales [11]. Usually, if one-dimensional length of fractal is magnified, the occupied space of fractal is then magnified by a noninteger power. This power is called the fractal dimension. For a fractal phenomenon or object, the character of similarity at different scales facilitates property characterization.

In an early work, Winslow reported the fractal nature of internal surface for cement paste [12]. Thereafter, the fractal characters in cement paste have been discussed a lot during past decades [13-20]. It was revealed that three types of fractal might be present in cement paste, that is, the pore mass fractal, the pore surface fractal, and the solid mass fractal [21]. Based on the fractal geometry theory, the complex pore structure in cement paste could be modeled via simple iterations, as done in literatures $[9,10,17,21]$. Moreover, the fractal geometry theory was able to account for various transport properties for porous materials such as permeability $[22,23]$ and electrical or thermal conductivity [24-28]. In this regard, the fractal modeling shows a great potential to well describe the complex pore structure as well as ionic diffusivity for cement based composites.

In this paper, pore structure in cement paste is modeled by means of the recently proposed solid mass fractal model [21]. Moreover, an enhanced Maxwell homogenization method that incorporates the solid mass fractal model is proposed to determine the associated ionic diffusivity. 

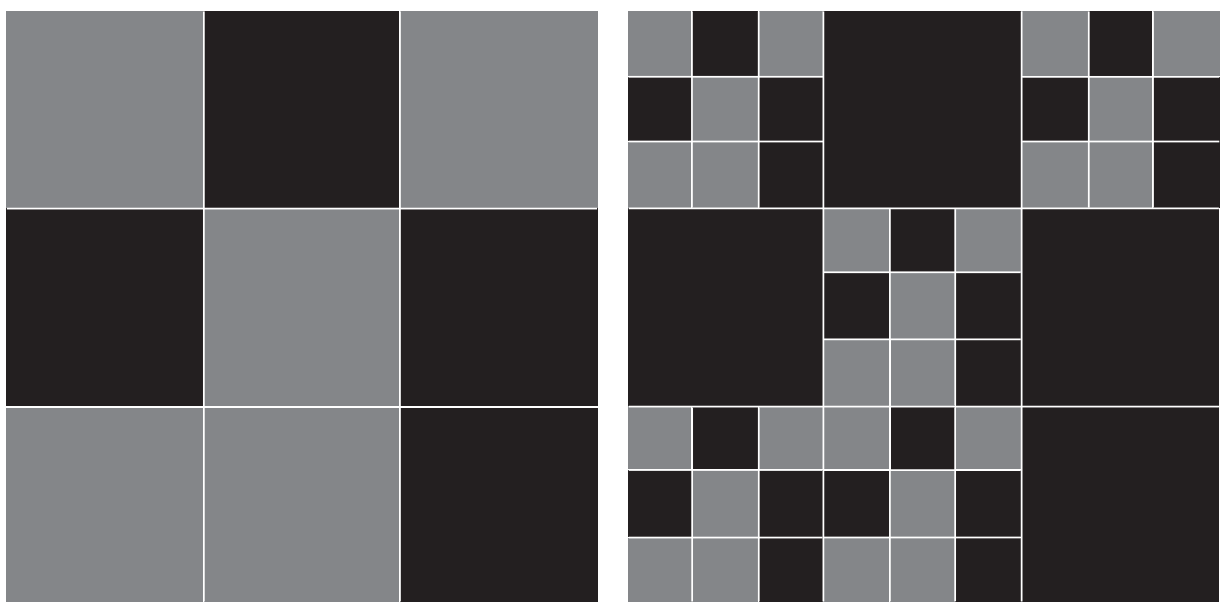

FIGURE 1: Schematic illustration of the solid mass fractal model with $E=2, n=3$, and $b=5$. Gray denotes the iterating phase and black denotes the pore phase.

Experiments are performed to validate the modeling, that is, mercury intrusion porosimetry (MIP) and rapid chloride migration (RCM).

\section{Solid Mass Fractal Model}

As shown in Figure 1, for the solid mass fractal to model porous medium, the generator refers to two distinct phases, that is, the pore phase and the iterating phase. At the initial step, a blank region with linear length $L$ is defined in $E$ dimension Euclidean space which can further be divided into $N$ identical subregions. Let $n$ be the number of subregions in each dimension such that $N=n^{E}$. Moreover, let $w$ and $b$, respectively, be the proportion and number of iterating phase in the generator such that $b=w N$. Upon successive iterations, the pattern of generator is repeated for the iterating phase at different scales. The generated pore structure can be analyzed as follows.

Step 1. The number of iterating phase and pore phase with the size $a_{1}=L / n$ reads $w N$ and $(1-w) N$, respectively.

Step 2. The number of iterating phase and pore phase with the size $a_{2}=L / n^{2}$ reads $(w N)^{2}$ and $(1-w) w N^{2}$, respectively.

Step $i$. The number of iterating phase and pore phase with the size $a_{i}=L / n^{i}$ reads $(w N)^{i}$ and $(1-w) N^{i} w^{(i-1)}$, respectively.

For the generated porous medium, the solid fraction or density $\chi$ is defined as the ratio of solid volume to the total volume that

$$
\chi=\frac{V_{\text {tot }}-V}{V_{\text {tot }}},
$$

where $V_{\text {tot }}$ is the total volume and $V$ is the resultant volume of pore phase. As a result, the solid fraction at Step i of iteration holds that $\chi_{i}=w^{i}$. If a constant $D=\log (N w) / \log (n)$ is defined, the solid fraction $\chi_{i}$ can then be rewritten as follows with respect to $a_{i}=L / n^{i}$ and $N=n^{E}$ :

$$
\chi_{i}=\left(\frac{a_{i}}{L}\right)^{E-D} .
$$

Equation (2) suggests that the generated porous medium is a solid mass fractal with the dimension of $D$. In a loglog diagram, the linearity shall be detected for the solid fraction $\chi_{i}$ against the pore size $a_{i}$. In addition, the pore size distribution in terms of $f$ versus $a_{i}$, that is, the cumulative porosity $f$ that counts from the large to the small pores, can be expressed as follows:

$$
f\left(\geq a_{i}\right)=1-\chi_{i}=1-\left(\frac{a_{i}}{L}\right)^{E-D} .
$$

\section{Modeling of Pore Structure}

The MIP test was performed to validate the solid mass fractal model [29]. Figure 2 shows the pore size distribution of cement paste with water cement (w/c) of 0.4 and age of 28 days, which was cured at the condition of $95 \% \pm 10 \%$ relative humidity and $20^{\circ} \mathrm{C} \pm 1^{\circ} \mathrm{C}$ temperature. For the solid fractal mass model, solid fraction is plotted against pore diameter in the log-log diagram, as shown in Figure 3. It can be noted that the sound piecewise linearity exists. Moreover, the slope of linearity varies within two pore ranges. Let $d_{\text {low }}$ and $d_{\text {upp }}$ be the lower and upper limit of pore diameter, and let $d_{\text {thr }}$ be the threshold of pore diameter for the two pore ranges. The values of $d_{\text {low }}, d_{\text {thr }}$, and $d_{\text {upp }}$ can be determined as $d_{\text {low }}=0.004 \mu \mathrm{m}$, $d_{\text {thr }}=0.1 \mu \mathrm{m}$, and $d_{\text {upp }}=2 \mu \mathrm{m}$. In other words, the two pore ranges, that is, the large and the small pore range, are located as $d_{\text {upp }} \sim d_{\text {thr }}$ and $d_{\text {thr }} \sim d_{\text {low }}$, that is, $2 \sim 0.1 \mu \mathrm{m}$ and $0.1 \sim 0.004 \mu \mathrm{m}$. The large pore range corresponds to capillary pores, while the small pore range corresponds to C-S-H gel pores.

With the determined pore ranges $\left(d_{\mathrm{upp}} \sim d_{\mathrm{thr}}, d_{\mathrm{thr}} \sim\right.$ $\left.d_{\text {low }}\right)$, the least square method is then applied to fit the associated slopes $\left(k_{\text {upp }}=0.0206, k_{\text {low }}=0.0607\right)$ as well as 


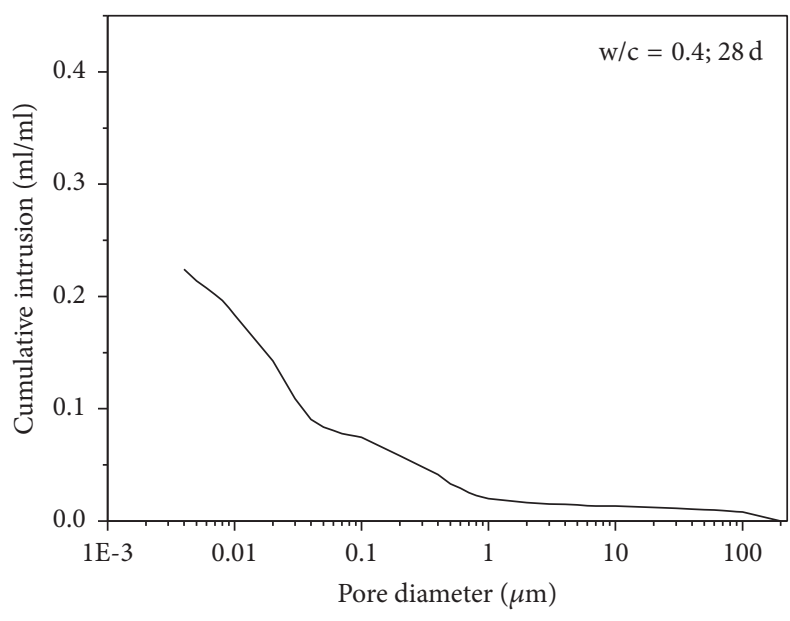

Figure 2: Porosimetric data example: cumulative intrusion volume against pore diameter.

the fractal dimensions $\left(D_{\text {upp }}=2.9794, D_{\text {low }}=2.9393\right)$ since $D_{\text {upp }}=E-k_{\text {upp }}, D_{\text {low }}=E-k_{\text {low }}$, and $E=3$. Meanwhile, it is possible to specify the partial porosity of large and small pore range $\left(f_{\text {upp }}=0.0746, f_{\text {low }}=0.1497\right)$ with the total porosity $f$ holding that $f=f_{\text {upp }}+f_{\text {low }}$. In rough comparison, the fractal dimension of small pore range is smaller than that of large pore range, that is, $D_{\text {low }}<D_{\text {upp }}$. The partial porosity of small pore range is larger than that of large pore range, that is, $f_{\text {low }}>f_{\text {upp }}$. The fractal modeling of pore structure in cement paste refers to two specific structures, that is, two fractal generators for the large and the small pore range.

For the large pore range, let two integers $i_{\text {upp }}$ and $n_{\text {upp }}$ be the step of iterations and the number of subregions such that $i_{\text {upp }}=\log \left(d_{\text {upp }} / d_{\text {thr }}\right) / \log \left(n_{\text {upp }}\right)$. The solid mass fractal model requires $n_{\text {upp }} \geq 2$, $i_{\text {upp }} \geq 2$, and $n_{\text {upp }} \cdot{ }^{\wedge} D_{\text {upp }} \geq 1$. In this paper, the symbol.$^{\wedge}$ denotes the power operator. Without complicated mathematical analysis, it leads to $n_{\text {upp }}=4$ and $i_{\text {upp }}=3$. The value of $w_{\text {upp }}$ is obtained via $\log \left(w_{\text {upp }}\right)=$ $\left(D_{\text {upp }}-3\right) \cdot \log \left(n_{\text {upp }}\right)$. The number of iterating phase $b_{\text {upp }}$ holds that $b_{\text {upp }}=w_{\text {upp }} \cdot\left(n_{\text {upp }}\right)^{3}$. The intrinsic porosity of generated pore structure $f_{\text {upp }}^{*}$ holds that $f_{\text {upp }}^{*}=1-\left[b_{\text {upp }} /\left(n_{\text {upp }}\right)^{3}\right] \cdot{ }^{\wedge} i_{\text {upp }}$. Details of analysis for the example are shown as follows:

The large pore range: $n_{\text {upp }}=4, i_{\text {upp }}=3$

The proportion of iterating phase: $w_{\mathrm{upp}}=n_{\mathrm{upp}} \cdot \wedge\left(D_{\mathrm{upp}}\right.$ $-3)=4 \cdot^{\wedge}(-0.0206)=0.9718$

The number of iterating phases: $b_{\text {upp }}=w_{\text {upp }} \cdot\left(n_{\text {upp }}\right)^{3}=$ $0.9718 * 44^{\wedge} 3=62$

The intrinsic porosity: $f_{\text {upp }}^{*}=1-\left[b_{\text {upp }} /\left(n_{\text {upp }}\right)^{3}\right] \cdot \wedge i_{\text {upp }}$ $=1-(62 / 64) \cdot{ }^{\wedge} 3=0.0909$

For the small pore range, let two integers $i_{\text {low }}$ and $n_{\text {low }}$ be the step of iterations and the number of subregions, which holds that $i_{\text {low }}=\log \left(d_{\text {thr }} / d_{\text {low }}\right) / \log \left(n_{\text {low }}\right)$. The solid mass fractal model requires $n_{\text {low }} \geq 2, i_{\text {low }} \geq 2$, and $n_{\text {low }} \cdot{ }^{\wedge} D_{\text {low }} \geq 1$. It leads to $n_{\text {low }}=3$ and $i_{\text {low }}=3$. The value of $w_{\text {low }}$ is obtained via $\log \left(w_{\text {low }}\right)=\left(D_{\text {low }}-3\right) \cdot \log \left(n_{\text {low }}\right)$. The number

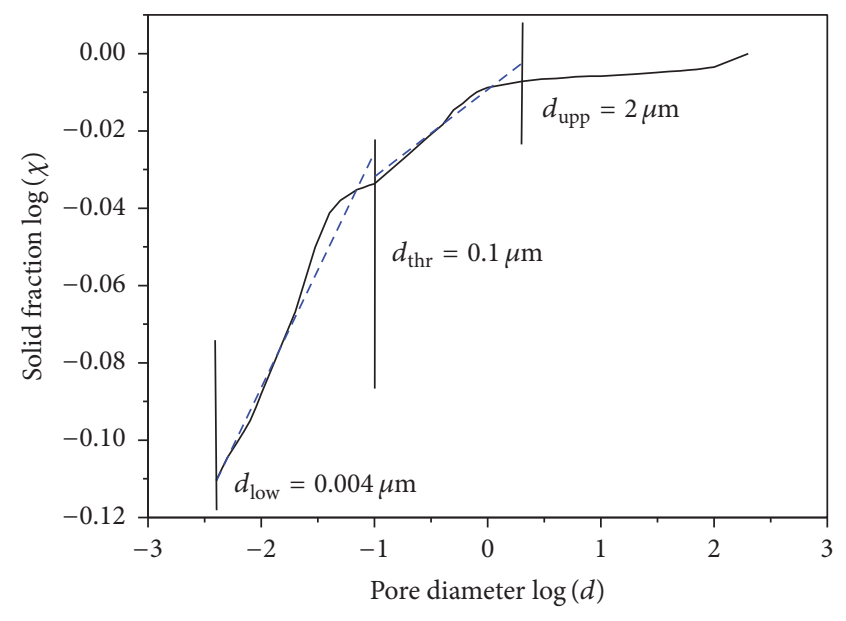

FIGURE 3: Parametric analysis: log-log diagram of solid fraction against pore diameter.

of iterating phase $b_{\text {low }}$ holds that $b_{\text {low }}=w_{\text {low }} \cdot\left(n_{\text {low }}\right)^{3}$. The intrinsic porosity of generated pore structure $f_{\text {low }}^{*}$ holds that $f_{\text {low }}^{*}=1-\left[b_{\text {low }} /\left(n_{\text {low }}\right)^{3}\right] \cdot{ }^{\wedge} i_{\text {low }}$. Details of analysis for the example are shown as follows:

The small pore range: $n_{\text {low }}=3, i_{\text {low }}=3$

The proportion of iterating phase: $w_{\text {low }}=n_{\text {low }} \cdot \wedge\left(D_{\text {low }}\right.$ $-3)=3 \cdot \wedge(-0.0607)=0.9355$

The number of iterating phases: $b_{\text {low }}=w_{\text {low }} \cdot\left(n_{\text {low }}\right)^{3}=$ $0.9355 * 3 \cdot^{\wedge} 3=25$

The intrinsic porosity: $f_{\text {low }}^{*}=1-\left[b_{\text {low }} /\left(n_{\text {low }}\right)^{3}\right] \cdot{ }^{\wedge} i_{\text {low }}=$ $1-(25 / 27) \cdot{ }^{\wedge} 3=0.2062$

The build-up of pore structure in cement paste follows from the large to the small pore range. In particular, after $i_{\text {upp }}$ steps of iteration to generate the large pore range, the size and number of iterating phase is $d_{\text {thr }}$ and $\left(b_{\text {upp }}\right) \cdot{ }^{\wedge} i_{\text {upp }}$, respectively. Note that during the process to generate the small pore range, not all the iterating phases with size of $d_{\mathrm{thr}}$ are to be further iterated but a proportion of $\gamma(\gamma \leq 1)$. In other words, the $1-\gamma$ proportion of iterating phases with size of $d_{\text {thr }}$ shall not be performed with further iterations. With respect to the chemical composition of cement paste, such $\gamma$ and $1-\gamma$ proportion of iterating phases are introduced as C-S$\mathrm{H}$ gel and solid phases, respectively. The solid phases may be anhydrous cement, calcium hydroxide crystal, Aft, and gypsum. It is necessary to determine $\gamma$ to fulfill the build-up of pore structure in cement paste. Herewith, consider the total porosity $f$ satisfying $f=f_{\text {upp }}^{*}+f_{\text {low }}^{*} \cdot \gamma \cdot\left(w_{\text {upp }}\right) \cdot{ }^{\wedge} i_{\text {upp }}$. Then, $f=0.2243, f_{\text {upp }}^{*}=0.0909, f_{\text {low }}^{*}=0.2062, w_{\text {upp }}=0.9718$, and $i_{\text {upp }}=3$ lead to $\gamma=0.7115$. Figure 4 shows one realization of modeled pore structure in cement paste. In particular, it refers to two specific structures, that is, the capillary structure and the C-S-H gel structure. The capillary structure of large pore range consists of three phases, that is, the (capillary) pore phase, the solid phase (anhydrous cement, calcium hydroxide crystal, AFt, and gypsum), and the C-S-H gel phase. The C-S$\mathrm{H}$ gel structure of small pore range consists of two phases, that 


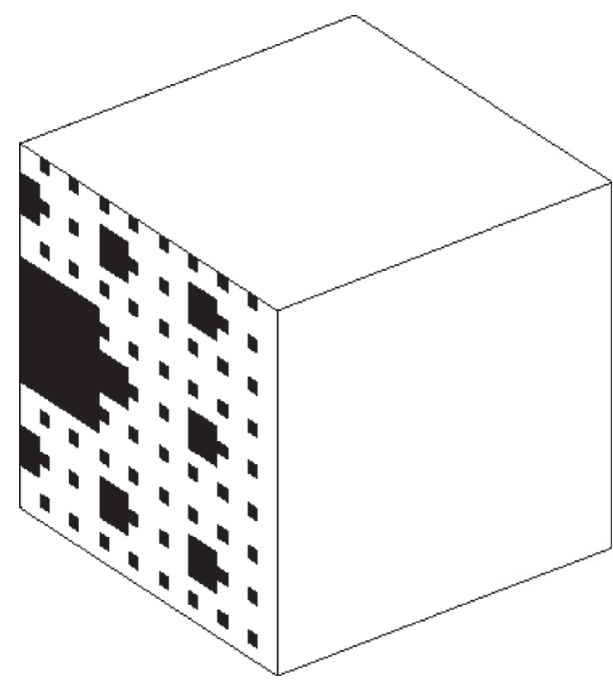

(a)

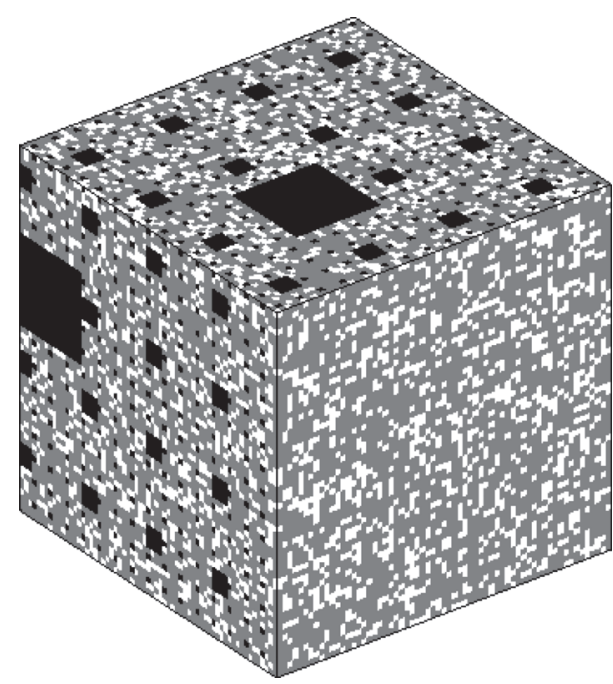

(b)

Figure 4: Realization of pore structure in cement paste (a) C-S-H gel structure $\left(n_{\text {low }}=3, i_{\text {low }}=3, b_{\text {low }}=25\right)$ with element size of $0.004 \mu$ m; (b) capillary structure of cement paste $\left(n_{\text {upp }}=4, i_{\text {upp }}=3, b_{\text {upp }}=62, \gamma=0.7115\right)$ with element size of $0.1 \mu \mathrm{m}$. Black denotes the pore phase, white denotes the solid phase, and gray denotes the C-S-H gel phase.

is, the (gel) pore phase and the solid phase (molecule layers within C-S-H gel). The pore size distribution can be written in terms of the piecewise form:

$$
\begin{aligned}
& \text { for } d>d_{\text {thr }}, d=d_{\text {upp }} /\left(n_{\text {upp }} \wedge j\right), f(\geq d)=1-w_{\text {upp }} \wedge{ }^{\wedge} j, \\
& j=1, \ldots, i_{\text {upp }} ; \\
& \text { for } d \leq d_{\text {thr }}, d=d_{\text {thr }} /\left(n_{\text {low }} \wedge{ }^{\prime} j\right), f(\geq d)=(1- \\
& \left.w_{\text {upp }} \wedge i_{\text {upp }}\right)+\left(1-w_{\text {low }} \wedge j\right) \cdot \gamma \cdot\left(w_{\text {upp }} \wedge i_{\text {upp }}\right), j= \\
& 1, \ldots, i_{\text {low }}
\end{aligned}
$$

In particular, for example, it holds that

$$
\begin{aligned}
& \text { for } d>0.1 \mu \mathrm{m}, d=d_{\mathrm{upp}} /\left(4^{\wedge} j\right), f(\geq d)=1- \\
& (62 / 64) \cdot{ }^{\wedge} j, j=1,2,3 \text {; } \\
& \text { for } d \leq 0.1 \mu \mathrm{m}, d=d_{\mathrm{thr}} /\left(3 \cdot^{\wedge} j\right), f(\geq d)=1- \\
& (62 / 64) \cdot{ }^{\wedge} 3+\left[1-(25 / 27) \cdot \wedge^{\wedge} j\right] \cdot \gamma \cdot(62 / 64) \cdot{ }^{\wedge} 3, j=1,2,3 .
\end{aligned}
$$

\section{Enhanced Maxwell Homogenization Method}

The calculation of effective properties of heterogeneous materials that contain inclusions of diverse shape and/or properties withstands a long history. Numerous homogenization schemes have been developed so far [30-32]. Among them, the Maxwell homogenization method is probably the oldest and also most famous one [33]. It considers heterogeneous materials with unknown effective ionic diffusivity $\left(D_{\text {eff }}\right)$ consisting of bulk phase with ionic diffusivity $\left(D_{B}\right)$ and inclusion phase with ionic diffusivity $\left(D_{I}\right)$. Mathematically, the Maxwell homogenization method can be described as follows:

$$
D_{\text {eff }}=\frac{D_{I}+2 D_{B}+2\left(D_{I}-D_{B}\right) c}{D_{I}+2 D_{B}-\left(D_{I}-D_{B}\right) c} D_{B},
$$

where $c$ is the volume fraction of inclusions.
An enhanced Maxwell homogenization method is proposed that incorporates the solid mass fractal model to calculate ionic diffusivity of cement paste. In particular, cement paste is viewed as the composite material consisting of two phases. One refers to the low-diffusivity C-S-H gel structure being bulk. The other refers to the high-diffusivity capillary structure being inclusions. The treatment of two distinct phases was also applied in the general effective media theory [34-36]. For bulk (C-S-H gel structure) and inclusion (capillary structure), the associated ionic diffusivity can be approximated in a power form of intrinsic porosity [37, 38]:

$$
\begin{aligned}
& D_{B}=\gamma D_{1}\left(f_{\mathrm{low}}^{*}\right)^{\delta_{1},} \\
& D_{I}=D_{2}\left(f_{\mathrm{upp}}^{*}\right)^{\delta_{2}},
\end{aligned}
$$

where $D_{1}$ and $D_{2}$ are constants, that is, ionic diffusivities in water corresponding to C-S-H gel and capillary structures. $\delta_{1}$ and $\delta_{2}$ are the cementation exponent in Archie's law for $\mathrm{C}-\mathrm{S}-\mathrm{H}$ gel and capillary structures. Other parameters, that is, $f_{\text {upp }}^{*}, f_{\text {low }}^{*}$, and $\gamma$ are defined in the solid mass fractal model. In addition, the volume fraction of inclusions is derived from

$$
c=\frac{f_{\text {upp }}}{f_{\text {upp }}+f_{\text {low }}} .
$$

As presented above, the Maxwell homogenization method is enhanced with critical parameters determined from the solid mass fractal model and Archie's law. Then, the enhanced Maxwell homogenization method can be applied to predict effective ionic diffusivity of cement paste.

\section{Rapid Chloride Migration Test}

The RCM test was performed to measure ionic (chloride) diffusivity of cement paste $[39,40]$. Cement paste samples 


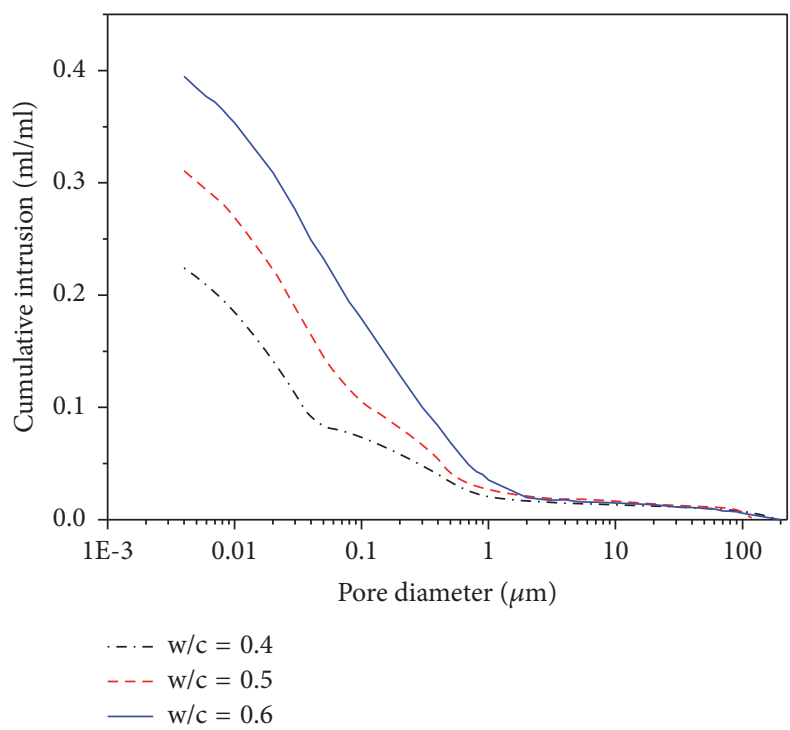

FIGURE 5: Porosimetric data of cement pastes with varying w/c ratios.

of $50 \mathrm{~mm}$ in thickness and $100 \mathrm{~mm}$ in diameter were prepared. Samples were then subjected to the vacuum saturation treatment. The saturated samples were treated to be surfacedry. Three samples of replicates were tested at the same time. Power sources with constant voltage outputs (adjustable in the range of $0 \sim 80 \mathrm{~V}$, accuracy of $0.05 \mathrm{~V}$ ) were applied. The used catholyte and anolyte were $10 \% \mathrm{NaCl}$ solution and $0.3 \mathrm{M} \mathrm{NaOH}$ solution, respectively. The electrolytes were refreshed after each series of tests. After the migration test, the samples were split and sprayed with a $0.1 \mathrm{M} \mathrm{AgNO}_{3}$ solution in order to determine the penetration depth of chlorides. The non-steady-state migration coefficient $D_{\mathrm{RCM}}$, that is, ionic (chloride) diffusivity, was calculated according to the NT Build 492 as follows:

$$
\begin{gathered}
D_{\mathrm{RCM}}=\frac{0.0239(273+T) l}{(U-2) t_{d}}\left(x_{d}\right. \\
-0.0238 \sqrt{\left.\frac{(273+T) l x_{d}}{U-2}\right),}
\end{gathered}
$$

where $D_{\mathrm{RCM}}$ is the non-steady-state migration coefficient, $\times 10^{-12} \mathrm{~m}^{2} / \mathrm{s}$; $U$ is absolute value of the applied voltage, $\mathrm{V} ; \mathrm{T}$ is average value of the initial and final temperatures in the anolyte solution, ${ }^{\circ} \mathrm{C} ; l$ is thickness of the specimen, $\mathrm{mm} ; x_{d}$ is average value of the penetration depth, $\mathrm{mm}$; and $t_{d}$ is the test duration, h.

\section{Results and Discussions}

Cement pastes with w/c ratios of $0.4,0.5$, and 0.6 were compared, as shown in Figure 5. All samples were cured at the same condition ( 28 days, $95 \% \pm 10 \%$ relative humidity, $20^{\circ} \mathrm{C} \pm 1^{\circ} \mathrm{C}$ temperature). Figure 6 shows the parametric analysis with respect to the solid mass fractal model. Results

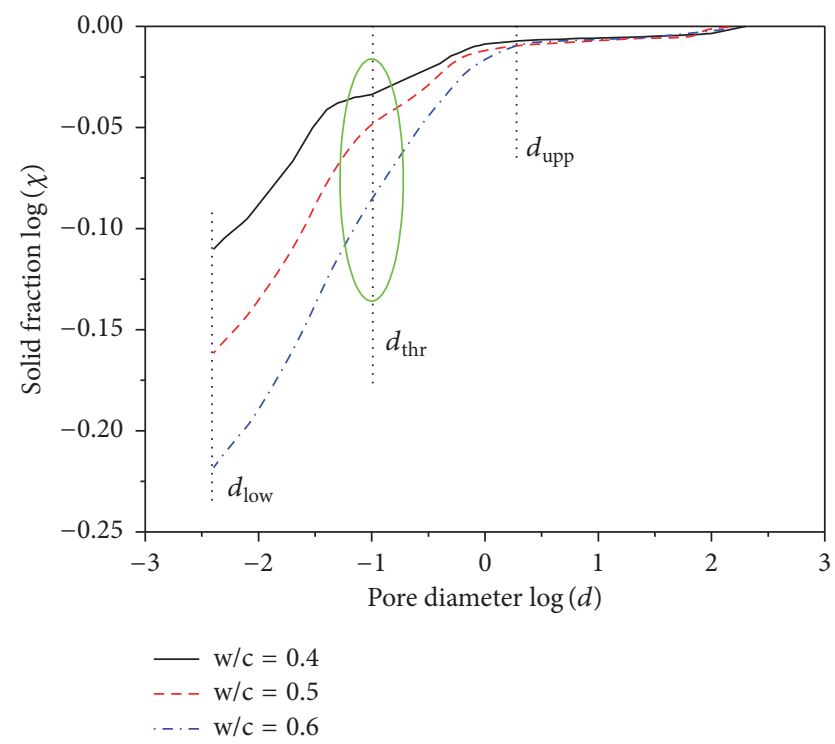

FIGURE 6: Parametric analysis of cement pastes with varying w/c ratios.

TABLE 1: Fractal dimensions and the associated porosities.

\begin{tabular}{lcccc}
\hline w/c ratio & $D_{\text {low }}$ & $f_{\text {low }}$ & $D_{\text {upp }}$ & $f_{\text {upp }}$ \\
\hline 0.4 & 2.9393 & 0.1497 & 2.9794 & 0.0746 \\
0.5 & 2.9089 & 0.1769 & 2.9701 & 0.1052 \\
0.6 & 2.9008 & 0.2161 & 2.9467 & 0.1789 \\
\hline
\end{tabular}

TABLE 2: Parameters of the solid mass fractal model.

\begin{tabular}{lccccccc}
\hline w/c ratio & $b_{\text {low }}$ & $n_{\text {low }}$ & $i_{\text {low }}$ & $\gamma$ & $b_{\text {upp }}$ & $n_{\text {upp }}$ & $i_{\text {upp }}$ \\
\hline 0.4 & 25 & & & 0.7115 & 62 & & \\
0.5 & 24 & 3 & 3 & 0.8123 & 62 & 4 & 3 \\
0.6 & 24 & & & 0.8928 & 60 & & \\
\hline
\end{tabular}

TABle 3: Parameters used for the enhanced Maxwell homogenization method.

\begin{tabular}{lccc}
\hline$D_{1}$ & $D_{2}$ & $\delta_{1}$ & $\delta_{2}$ \\
\hline $1.25 \times 10^{-10} \mathrm{~m}^{2} / \mathrm{s}$ & $1.25 \times 10^{-9} \mathrm{~m}^{2} / \mathrm{s}$ & 1.8 & 1.5 \\
\hline
\end{tabular}

are summarized in Tables 1 and 2. Table 1 suggests that as w/c ratio increases, the partial porosities $\left(f_{\text {upp }}, f_{\text {low }}\right)$ increase, while the fractal dimensions $\left(D_{\text {upp }}, D_{\text {low }}\right)$ decrease. Table 2 suggests that some parameters, that is, $n_{\text {low }}, i_{\text {low }}, n_{\text {upp }}$, and $i_{\text {upp }}$, do not change with w/c ratio, while some parameters, that is, $b_{\text {upp }}, b_{\text {low }}$, and $\gamma$, do. That means the three parameters $\left(b_{\text {upp }}, b_{\text {low }}, \gamma\right)$ are critical to characterize the effect of w/c ratio on geometry of capillary and C-S-H gel structure. Figure 7 shows the comparison of modeled and measured pore size distributions of cement pastes.

With the enhanced Maxwell homogenization method, ionic diffusivity of cement paste can be obtained. The parameters listed in Tables 1 and 2 are used, that is, $\left(f_{\text {upp }}, f_{\text {low }}\right)$ and $\left(n_{\text {low }}, i_{\text {low }}, n_{\text {upp }}, i_{\text {upp }}, b_{\text {upp }}, b_{\text {low }}, \gamma\right)$. Besides, values of the parameters $\left(D_{1}, D_{2}, \delta_{1}, \delta_{2}\right)$ are suggested in Table 3 . In 


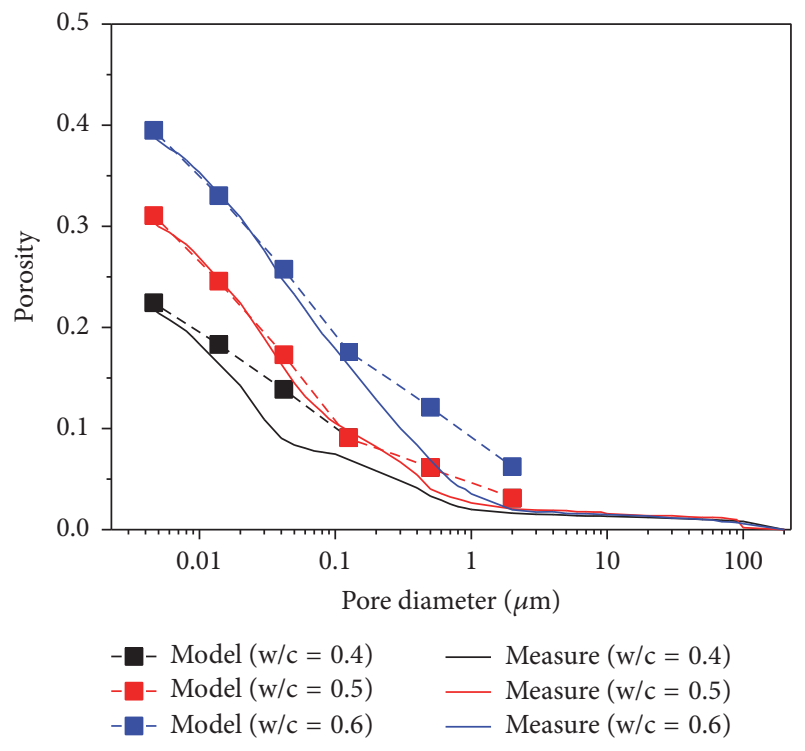

FIGURE 7: Modeled and measured pore size distribution of cement pastes with varying $\mathrm{w} / \mathrm{c}$ ratios.

TABLE 4: Parameters measured in RCM test of cement pastes.

\begin{tabular}{lccccc}
\hline w/c ratio & $T\left({ }^{\circ} \mathrm{C}\right)$ & $U(\mathrm{~V})$ & $t_{d}$ (hour) & $l(\mathrm{~mm})$ & $x_{d}(\mathrm{~mm})$ \\
\hline 0.4 & 26.0 & 25 & 24 & 49.6 & 18.7 \\
0.5 & 25.1 & 15 & 24 & 49.8 & 18.9 \\
0.6 & 23.5 & 15 & 24 & 50.2 & 29.5 \\
\hline
\end{tabular}

particular, $D_{1}=1.25 \times 10^{-10} \mathrm{~m}^{2} / \mathrm{s}$ is empirical. It leads to the ionic (chloride) diffusivity of $\mathrm{C}-\mathrm{S}-\mathrm{H}$ gel around $8 \times$ $10^{-12} \mathrm{~m}^{2} / \mathrm{s}$, which is comparable to the value suggested in [41]. $D_{2}=1.25 \times 10^{-9} \mathrm{~m}^{2} / \mathrm{s}$ is taken after [42]. The cementation exponents of $\delta_{1}=1.8$ and $\delta_{2}=1.5$ for C-S-H gel and capillary structures are suggested from extensive tests, which differs from the usual range of 1.8-2.0 for consolidated sandstones [37].

For the RCM test, the measured parameters are listed in Table 4, which leads to an easy calculation of the non-steadystate migration coefficient $D_{\mathrm{RCM}}$. As shown in Figure 8, results of the fractal modeling $\left(D_{\text {eff }}\right)$ agree well with those obtained from experiment $\left(D_{\mathrm{RCM}}\right)$. In this regard, the present fractal modeling provides an optional description of pore structure as well as ionic diffusivity for cement paste.

\section{Concluding Remarks}

In this paper, modeling of pore structure as well as ionic diffusivity for cement paste is performed based on the fractal geometry theory. The solid mass fractal model is applied to model pore structure, and an enhanced Maxwell homogenization method that incorporates the solid mass fractal model is proposed to calculate the associated ionic diffusivity. Some general conclusions are drawn as follows:

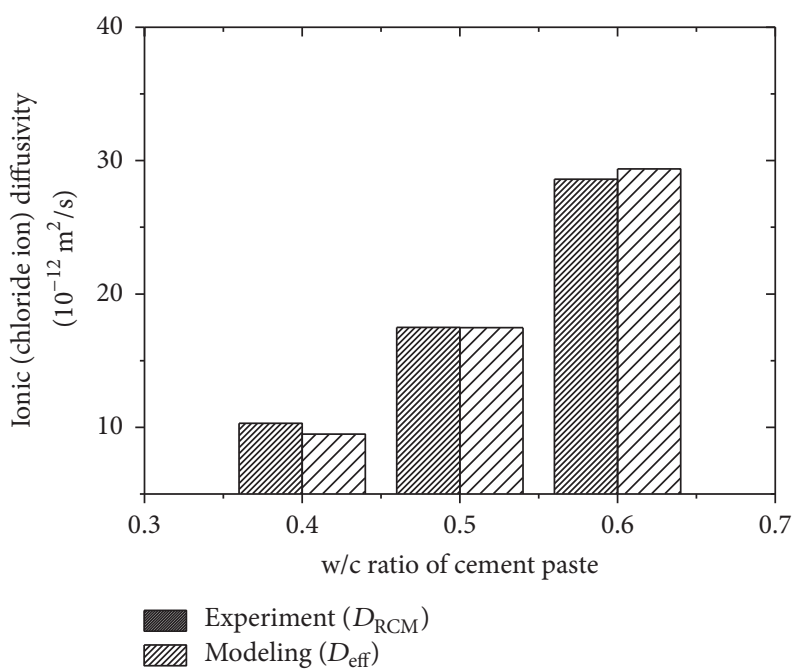

FIGURE 8: Modeled and measured ionic diffusivity of cement pastes with varying $\mathrm{w} / \mathrm{c}$ ratios.

(1) The solid mass fractal model well describes pore structure in cement paste in terms of two pore ranges, that is, the large (capillary) and the small (C-S-H gel) pore range. The fractal generator varies with the pore range.

(2) The enhanced Maxwell homogenization method considers cement paste consisting of low-diffusivity C-S$\mathrm{H}$ gel structure and high-diffusivity capillary structure. Archie's law is applicable to approximate ionic diffusivity for C-S-H gel and capillary structures.

\section{Nomenclature}

$L:$

$E$ :

$N$ :

$n$ :

$w:$

$b:$

$i$ :

$a_{i}:$

$\chi:$

$V_{\text {tot }}:$

$V:$

$D:$

$f$ : $\quad$ Cumulative porosity $\mathrm{f}$ that counts from

large to small pore

$d: \quad$ Equivalent pore diameter

$d_{\text {low }}, d_{\text {thr }}, d_{\text {upp }}$ : Lower, threshold, and upper limit of equivalent pore diameter

$k_{\text {upp }}, k_{\text {low }}$ : $\quad$ Slope of large and small pore range

$D_{\text {upp }}, D_{\text {low }}$ :

Linear length of modeling space

Dimension of Euclidean space

Total number of identical subregions dimension

Proportion of iterating phase in generator

Number of iterating phases in generator

Total volume of porous medium

Volume of pore phase

Fractal dimension

Fractal dimension of large and small pore range 


\begin{tabular}{|c|c|}
\hline$f_{\text {upp }}, f_{\text {low }}:$ & $\begin{array}{l}\text { Partial porosity of large and small } \\
\text { pore range }\end{array}$ \\
\hline$i_{\text {upp }}, i_{\text {low }}:$ & $\begin{array}{l}\text { Step of iteration to generate large and } \\
\text { small pore range }\end{array}$ \\
\hline$n_{\text {upp }}, n_{\text {low }}:$ & $\begin{array}{l}\text { One-dimension number of } \\
\text { subregions for large and small pore } \\
\text { range }\end{array}$ \\
\hline$w_{\text {upp }}, w_{\text {low }}:$ & $\begin{array}{l}\text { Proportion of iterating phase in } \\
\text { generator for large and small pore } \\
\text { range }\end{array}$ \\
\hline$b_{\text {upp }}, b_{\text {low }}:$ & $\begin{array}{l}\text { Number of iterating phases in } \\
\text { generator for large and small pore } \\
\text { range }\end{array}$ \\
\hline$f_{\text {upp' }}^{*}, f_{\text {low }}^{*}:$ & $\begin{array}{l}\text { Intrinsic porosity of large and small } \\
\text { pore range }\end{array}$ \\
\hline$\gamma:$ & $\begin{array}{l}\text { Proportion of iterating phase being } \\
\text { C-S-H gel }\end{array}$ \\
\hline$D_{\text {eff }}:$ & $\begin{array}{l}\text { Effective ionic diffusivity of porous } \\
\text { medium }\end{array}$ \\
\hline$D_{B}, D_{I}:$ & $\begin{array}{l}\text { Ionic diffusivity of bulk and inclusion } \\
\text { phase }\end{array}$ \\
\hline$c:$ & Volume fraction of inclusions \\
\hline$D_{1}, D_{2}:$ & $\begin{array}{l}\text { Ionic diffusivity in water } \\
\text { corresponding to gel and capillary } \\
\text { pore }\end{array}$ \\
\hline$\delta:$ & $\begin{array}{l}\text { Cementation exponent in Archie's } \\
\text { law }\end{array}$ \\
\hline$D_{\mathrm{RCM}}:$ & $\begin{array}{l}\text { Non-steady-state migration } \\
\text { coefficient }\end{array}$ \\
\hline$U:$ & Absolute value of the applied voltage \\
\hline$T:$ & $\begin{array}{l}\text { Average value of the initial and final } \\
\text { temperatures in anolyte solution }\end{array}$ \\
\hline$l:$ & Thickness of the specimen \\
\hline$x_{d}:$ & $\begin{array}{l}\text { Average value of the penetration } \\
\text { depth }\end{array}$ \\
\hline$t_{d}$ & Test duration. \\
\hline
\end{tabular}

\section{Competing Interests}

The authors declare that they have no competing interests.

\section{Acknowledgments}

The authors gratefully acknowledge the financial support from Key Basic Research Project (Grant No. 2015CB655105), State Key Laboratory for Track Technology of High-speed Railway (Grant no. 2014G004-F), and Jiangsu Natural Science Foundation (Grant no. BK20150621).

\section{References}

[1] L. Tang, "Electrically accelerated methods for determining chloride diffusivity in concrete-current development," Magazine of Concrete Research, vol. 48, no. 176, pp. 173-179, 1996.

[2] E. J. Garboczi and D. P. Bentz, "Multiscale analytical/numerical theory of the diffusivity of concrete," Advanced Cement Based Materials, vol. 8, no. 2, pp. 77-88, 1998.

[3] H. M. Jennings, J. W. Bullard, J. J. Thomas, J. E. Andrade, J. J. Chen, and G. W. Scherer, "Characterization and modeling of pores and surfaces in cement paste: correlations to processing and properties," Journal of Advanced Concrete Technology, vol. 6, no. 1, pp. 5-29, 2008.

[4] K. Maekawa, T. Ishida, and T. Kishi, "Multiscale modeling of concrete performance integrated material and structural mechanics," Journal of Advanced Concrete Technology, vol. 1, no. 2, pp. 91-126, 2003.

[5] G. W. Sun, W. Sun, Y. S. Zhang, and Z. Y. Liu, "Prediction of the effective diffusion coefficient of chloride ions in cement-based composite materials," Journal of Materials in Civil Engineering, vol. 24, no. 9, pp. 1245-1253, 2012.

[6] D. Giménez, E. Perfect, W. J. Rawls, and Y. Pachepsky, "Fractal models for predicting soil hydraulic properties: a review," Engineering Geology, vol. 48, no. 3-4, pp. 161-183, 1997.

[7] J. L. Pérez Bernal and M. A. Bello, "Fractal geometry and mercury porosimetry. Comparison and application of proposed models on building stones," Applied Surface Science, vol. 185, no. 1-2, pp. 99-107, 2001.

[8] M. Arandigoyen, J. L. Perez Bernal, M. A. Bello López, and J. I. Alvarez, "Lime-pastes with different kneading water: pore structure and capillary porosity," Applied Surface Science, vol. 252, no. 5, pp. 1449-1459, 2005.

[9] C. Atzeni, G. Pia, and U. Sanna, "Fractal modelling of mediumhigh porosity $\mathrm{SiC}$ ceramics," Journal of the European Ceramic Society, vol. 28, no. 14, pp. 2809-2814, 2008.

[10] C. Atzeni, G. Pia, U. Sanna, and N. Spanu, "A fractal model of the porous microstructure of earth-based materials," Construction and Building Materials, vol. 22, no. 8, pp. 1607-1613, 2008.

[11] B. B. Mandelbrot, P. Pfeifer, O. Biham, O. Malcai, D. A. Lidar, and D. Avnir, "Is nature fractal?" Science, vol. 279, no. 5352, pp. 783-786, 1998.

[12] D. N. Winslow, "The fractal nature of the surface of cement paste," Cement and Concrete Research, vol. 15, no. 5, pp. 817-824, 1985.

[13] D. Pearson and A. J. Allen, "A study of ultrafine porosity in hydrated cements using small angle neutron scattering," Journal of Materials Science, vol. 20, no. 1, pp. 303-315, 1985.

[14] M. Kriechbaum, G. Degovics, J. Tritthart, and P. Laggner, "Fractal structure of portland cement paste during age hardening analyzed by small-angle X-ray scattering," Progress in Colloid and Polymer Science, vol. 79, pp. 101-105, 1989.

[15] D. A. Lange, H. M. Jennings, and S. P. Shah, "Image analysis techniques for characterization of pore structure of cementbased materials," Cement and Concrete Research, vol. 24, no. 5, pp. 841-853, 1994.

[16] D. N. Winslow, J. M. Bukowski, and J. F. Young, "The fractal arrangement of hydrated cement paste," Cement and Concrete Research, vol. 25, no. 1, pp. 147-156, 1995.

[17] X. Ji, S. Y. N. Chan, and N. Feng, "Fractal model for simulating the space-filling process of cement hydrates and fractal dimensions of pore structure of cement-based materials," Cement and Concrete Research, vol. 27, no. 11, pp. 1691-1699, 1997.

[18] A. Heinemann, H. Hermann, and F. Häussler, "SANS analysis of fractal microstructures in hydrating cement paste," Physica B: Condensed Matter, vol. 276-278, pp. 892-893, 2000.

[19] Y. Wang and S. Diamond, "A fractal study of the fracture surfaces of cement pastes and mortars using a stereoscopic SEM method," Cement and Concrete Research, vol. 31, no. 10, pp. 13851392, 2001.

[20] Q. Zeng, K. F. Li, T. Fen-Chong, and P. Dangla, "Surface fractal analysis of pore structure of high-volume fly-ash cement pastes," Applied Surface Science, vol. 257, no. 3, pp. 762-768, 2010. 
[21] Y. Gao, K. Wu, and J. Jiang, "Examination and modeling of fractality for pore-solid structure in cement paste: starting from the mercury intrusion porosimetry test," Construction and Building Materials, vol. 124, pp. 237-243, 2016.

[22] T. J. Miao, B. Yu, Y. G. Duan, and Q. T. Fang, "A fractal analysis of permeability for fractured rocks," International Journal of Heat and Mass Transfer, vol. 81, pp. 75-80, 2015.

[23] S. Wang, T. Wu, H. Qi, Q. Zheng, and Q. Zheng, "A permeability model for power-law fluids in fractal porous media composed of arbitrary cross-section capillaries," Physica A, vol. 437, pp. 1220, 2015.

[24] G. Pia and U. Sanna, "Intermingled fractal units model and electrical equivalence fractal approach for prediction of thermal conductivity of porous materials," Applied Thermal Engineering, vol. 61, no. 2-3, pp. 186-192, 2013.

[25] W. Wei, J. Cai, X. Hu, and Q. Han, "An electrical conductivity model for fractal porous media," Geophysical Research Letters, vol. 42, no. 12, pp. 4833-4840, 2015.

[26] H.-Q. Jin, X.-L. Yao, L.-W. Fan, X. Xu, and Z.-T. Yu, “Experimental determination and fractal modeling of the effective thermal conductivity of autoclaved aerated concrete: effects of moisture content," International Journal of Heat and Mass Transfer, vol. 92, pp. 589-602, 2016.

[27] S. Negrelli, R. P. Cardoso, and C. J. Hermes, "A finite-volume diffusion-limited aggregation model for predicting the effective thermal conductivity of frost," International Journal of Heat and Mass Transfer, vol. 101, pp. 1263-1272, 2016.

[28] G. Pia, "High porous yttria-stabilized zirconia with aligned pore channels: morphology directionality influence on heat transfer," Ceramics International, vol. 42, no. 10, pp. 11674-11681, 2016.

[29] R. A. Cook and K. C. Hover, "Mercury porosimetry of hardened cement pastes," Cement and Concrete Research, vol. 29, no. 6, pp. 933-943, 1999.

[30] R. W. Zimmerman, "Elastic moduli of a solid containing spherical inclusions," Mechanics of Materials, vol. 12, no. 1, pp. 17-24, 1991.

[31] M. Kachanov, I. Tsukrov, and B. Shafiro, "Effective moduli of solids with cavities of various shapes," Applied Mechanics Reviews, vol. 47, no. 1, pp. 151-174, 1994.

[32] S. K. Kanaun and D. Jeulin, "Elastic properties of hybrid composites by the effective field approach," Journal of the Mechanics and Physics of Solids, vol. 49, no. 10, pp. 2339-2367, 2001.

[33] I. Sevostianov and A. Giraud, "Generalization of Maxwell homogenization scheme for elastic material containing inhomogeneities of diverse shape," International Journal of Engineering Science, vol. 64, pp. 23-36, 2013.

[34] L. Cui and J. H. Cahyadi, "Permeability and pore structure of OPC paste," Cement and Concrete Research, vol. 31, no. 2, pp. 277-282, 2001.

[35] J. Zhang and Z. J. Li, "Application of GEM equation in microstructure characterization of cement-based materials," Journal of Materials in Civil Engineering, vol. 21, no. 11, pp. 648656, 2009.

[36] J. Zhao, J.-J. Zheng, and G.-F. Peng, "An effective medium approach for predicting the intrinsic permeability of heated cement paste," Advances in Cement Research, vol. 27, no. 4, pp. 240-246, 2015.

[37] G. E. Archie, "Introduction to petrophysics of reservoir rocks," American Association of Petroleum Geologists Bulletin, vol. 34, no. 5, pp. 943-961, 1950.
[38] H. S. Salem and G. V. Chilingarian, "The cementation factor of Archie's equation for shaly sandstone reservoirs," Journal of Petroleum Science and Engineering, vol. 23, no. 2, pp. 83-93, 1999.

[39] L. Tang and L. Nilsson, "Rapid determination of the 317 chloride diffusivity in concrete by applying an electric field," $A C I$ Materials Journal, vol. 89, no. 1, pp. 49-53, 1992.

[40] Z. Yu and G. Ye, "New perspective of service life prediction of fly ash concrete," Construction and Building Materials, vol. 48, pp. 764-771, 2013.

[41] E. J. Garboczi and D. P. Bentz, "Computer simulation of the diffusivity of cement-based materials," Journal of Materials Science, vol. 27, no. 8, pp. 2083-2092, 1992.

[42] S. Kerisit and C. X. Liu, "Molecular simulations of water and ion diffusion in nanosized mineral fractures," Environmental Science and Technology, vol. 43, no. 3, pp. 777-782, 2009. 

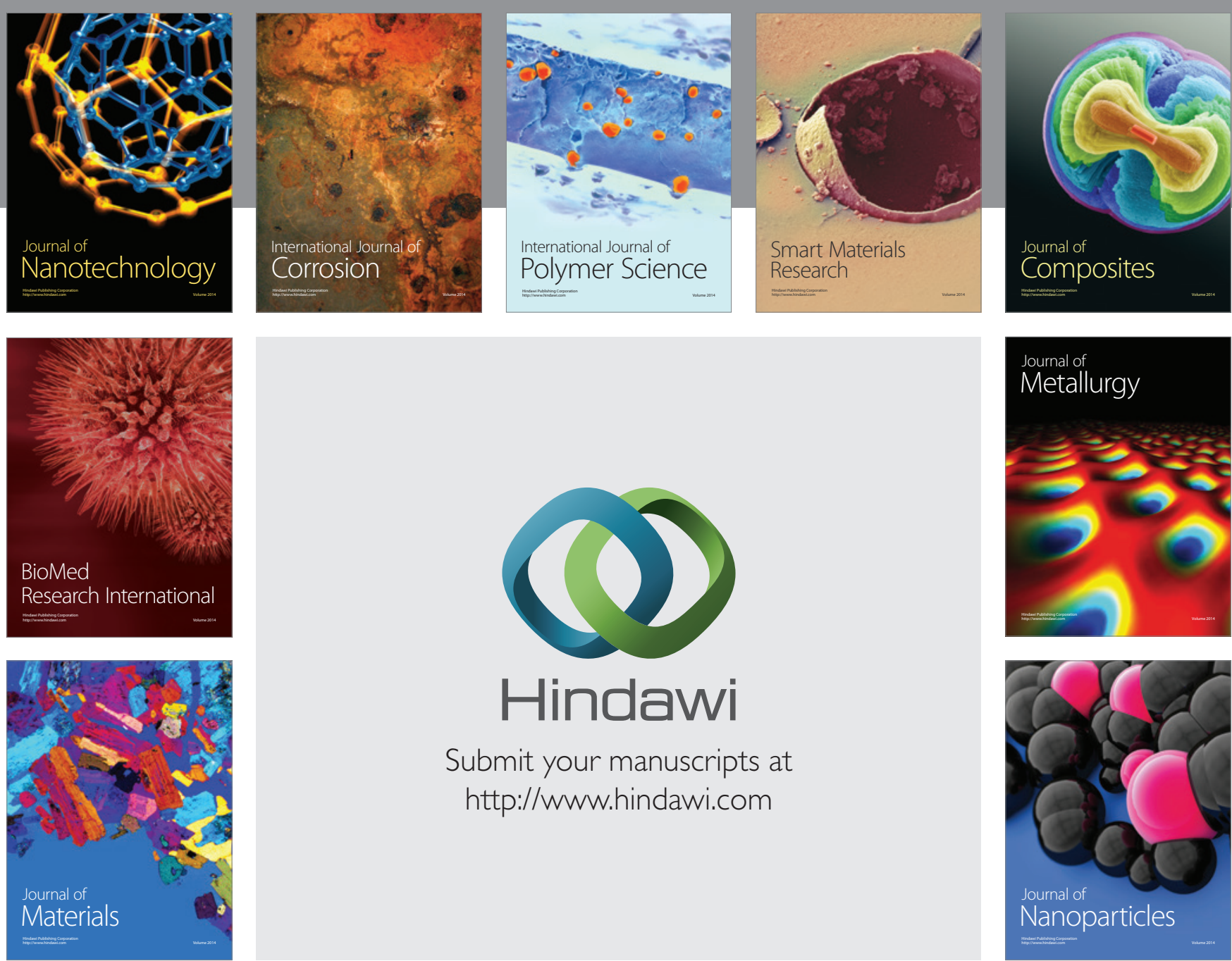

\section{Hindawi}

Submit your manuscripts at

http://www.hindawi.com

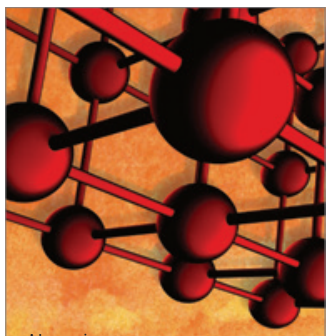

Materials Science and Engineering
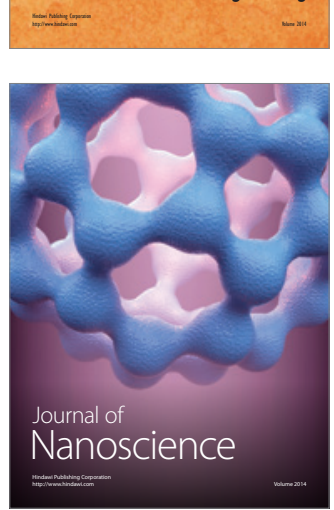
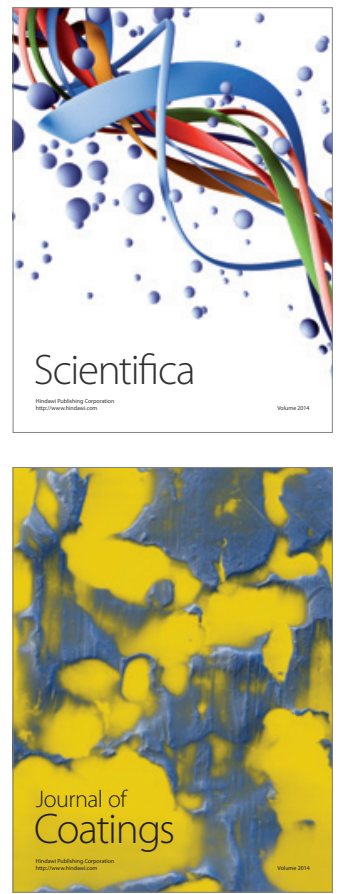
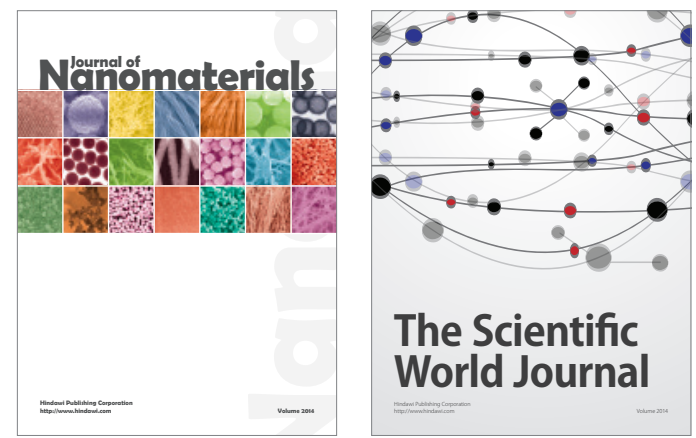

The Scientific World Journal
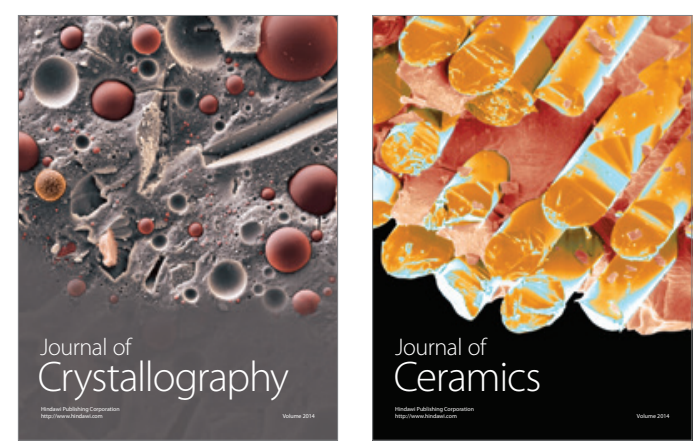
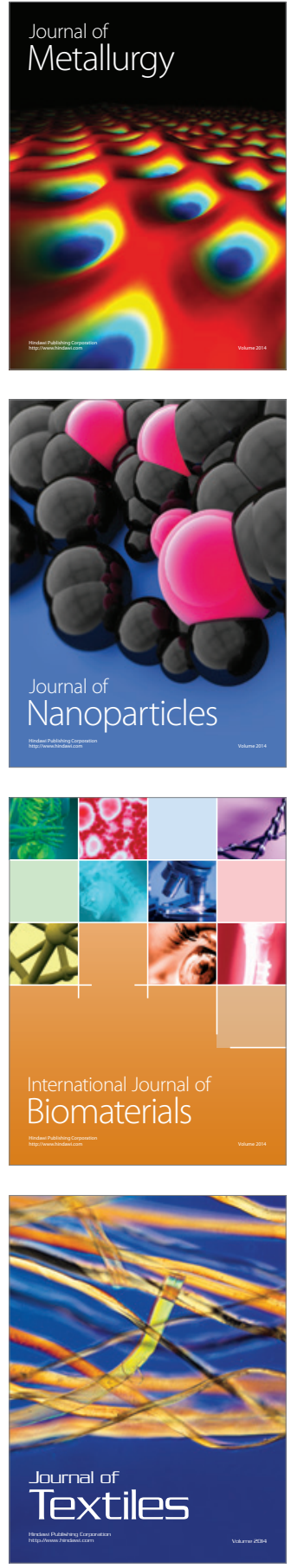